Research Article

\title{
Determinants of Intraocular Pressure (IOP) of Glaucoma Patients at Felege Hiwot Referral Hospital, Bahir Dar, Ethiopia
}

\author{
Mitiku Wale Muluneh (iD) and Shewayiref Geremew Gebremichael \\ Department of Statistics, Faculty of Natural and Computational Sciences, Debre Tabor University, Debre Tabor, Ethiopia \\ Correspondence should be addressed to Mitiku Wale Muluneh; mitikuwale@gmail.com
}

Received 24 April 2021; Accepted 1 September 2021; Published 20 September 2021

Academic Editor: Carol J. Burns

Copyright (c) 2021 Mitiku Wale Muluneh and Shewayiref Geremew Gebremichael. This is an open access article distributed under the Creative Commons Attribution License, which permits unrestricted use, distribution, and reproduction in any medium, provided the original work is properly cited.

Background. Glaucoma is a leading cause of irreversible blindness in the world associated with characteristic damage to the optic nerve and patterns of visual field loss due to retinal ganglion cell degeneration. The main objective of this study was to investigate determinants for the variation of intraocular pressure of glaucoma patients under treatment at Felege Hiwot Referral Hospital, Bahir Dar, Ethiopia. Methods. A retrospective cohort study design was conducted on 328 randomly selected glaucoma patients in the ophthalmology clinic at the hospital under the follow-up period from January 2014 to December 2018. Glaucoma patients who have two and more than two visits in the study period were included in the study, but patients who are attending medications less than two visits were excluded from the study. A linear mixed-effects model for intraocular pressure change was used for data analysis. Result. The estimated coefficient of fixed effect intercept was 25.1829, which indicates that the average IOP of the patients was $25.1829 \mathrm{mmHg}$ at baseline time by excluding all covariates in the model ( $p$ value $<0.0001$ ), age $(\beta=0.07,95 \% \mathrm{CI} 0.03,0.11)$, urban residence $(\beta=-1.60,95 \% \mathrm{CI}-2.84,-0.36)$, family history of glaucoma $(\beta=4.90,95 \% \mathrm{CI} 3.38-6.43)$, timolol and pilocarpine medication $(\beta=-2.02,95 \% \mathrm{CI}-4.01,-0.03)$, cup-disk ratio $>0.7(\beta=2.60,95 \%$ CI 1.24-3.96), and follow-up time $(\beta=-0.34,95 \%$ CI $-0.47,-0.21)$ were significantly associated with intraocular pressure of glaucoma patients. Conclusion. The predictor age, residence, family history of glaucoma, type of medication, cup-disk ratio, and follow-up time were significantly associated with the intraocular pressure of glaucoma patients. Therefore, healthcare providers give more attention and prioritize those identified factors and give frequent counseling about reducing intraocular pressure of glaucoma patients.

\section{Introduction}

Glaucoma is a leading cause of irreversible blindness in the world associated with characteristic damage to the optic nerve and patterns of visual field loss due to retinal ganglion cell degeneration [1]. Glaucoma encompasses a group of ophthalmic diseases that are believed to share the common pathophysiology of elevated intraocular pressure, or abnormal sensitivity to high normal intraocular pressure, resulting in damage to the nerve fiber layer of the retina and irreversible visual loss [2]. IOP is the fluid pressure inside the eye, and eye-care professionals use a tonometer to determine this. Most tonometers are calibrated to measure pressure in millimeters of mercury $(\mathrm{mmHg})$ [3].

Ocular hypertension refers to a situation within which the pressure inside the eye is beyond the statistical normal.
Normal eye pressure ranges from 10 to $21 \mathrm{mmHg}$. However, ocular hypertension is eye pressure greater than $21 \mathrm{mmHg}$. IOP measurement is an important aspect in the evaluation of patients at risk from glaucoma. The increase in pressure can damage the optic nerve and will cause loss of vision. Elevated intraocular pressure is a major risk factor for the development or progression of glaucoma, and IOP reduction is a well-known treatment strategy for slowing the progression of the disease [4]. IOP reduction by medical, laser, and surgical treatments remain the only clinically proven treatment of glaucoma.

In Africa, glaucoma is the second leading cause of blindness, and approximately $15 \%$ of blindness in the Africa continent is due to glaucoma. In many regions of subSaharan Africa, the ratio of an ophthalmologist to the patient is one in one million $(1: 1000000)$ people. The shortage of 
trained eye-care professionals is linked to a limited number of eye hospitals, and the majority of eye hospitals are found in urban centers. According to the Tema Eye Survey in Ghana, the overall prevalence of primary open-angle glaucoma is $6.8 \%$ for people over 40 years of age and increased to $14.6 \%$ for people over 80 years of age. Therefore, Ghana is a leading country with the highest glaucoma prevalence rate in subSaharan Africa where about 35000 patients are blind from 700,000 cases; it indicates that, for every 20 individuals, at least 1 individual lives with glaucoma [5].

In Ethiopia, glaucoma disease is the fifth most common cause of blindness, and 62,000 people in 2006 were living with the case [6]. Due to the different conditions such as inadequate information of public healthcare, inaccessible eye-care services, and low level of public awareness, glaucoma patients come for help after they have become either unilaterally or bilaterally blind [7]. By understanding the abovementioned conditions, not a study was yet undertaken on glaucoma patients in Ethiopia; therefore, the study aimed at identifying the determinant factors of IOP in glaucoma patients in the Ophthalmology Clinic of Felege Hiwot Referral Hospital, North West Ethiopia.

\section{Methods}

2.1. Study Design and Settings. A retrospective cohort study design was employed to retrieve relevant information from the medical records/charts of glaucoma patients. This study was conducted at Felege Hiwot Referral Hospital (FHRH), Bahir Dar, North West Ethiopia. Bahir Dar is the capital city of Amhara National Regional State. It is located $563 \mathrm{~km}$ far from Addis Ababa. This hospital serves as a referral hospital for people who came from different surrounding areas. The ophthalmologist measured the IOP of 328 glaucoma patients. IOP measurements were made with the Goldmann applanation tonometer (GAT).

2.2. Sources of Population. The glaucoma patients were the source of the population for this study. The data were collected from the medical charts of glaucoma patients in the ophthalmology clinic at FHRH under the follow-up from January 2014 to December 2018.

\subsection{Inclusion and Exclusion Criteria}

2.3.1. Inclusion Criteria. Glaucoma patients who have two and more than two visits in the study period from medical charts were included in the study. The total numbers of glaucoma patients who fulfill the inclusion criteria are 328 .

2.3.2. Exclusion Criteria. Patients who were attending medications for less than two visits were excluded from the study.

\subsection{Study Variables}

2.4.1. Dependent Variable. The dependent variable was IOP which is measured in millimeters of mercury ( $\mathrm{mmHg}$ ) for every six months irrespective of patient visits to the ophthalmology clinic of the hospital, and a patient with full follow-ups had 11 visits including the baseline. The dependent variable is continuous.

2.4.2. Independent Variables. The independent variables were gender (female and male), place of residence (rural and urban), age in years, family history with glaucoma (yes and no), family support (yes and no), type of medication (timolol, timolol with pilocarpine, timolol with diamox, and timolol with diamox with pilocarpine), duration of treatment (less than 2 years, 2-5 years, and above 5 years), stage of glaucoma (early, moderate, and advanced), and cup-disc ratio $(\leq 0.7,>0.7)$.

2.5. Operational Definition. Early glaucoma: the presence of glaucomatous optic nerve head damage but no visual field abnormalities within the central $10^{\circ}$ and cup-to-disc ratio $(\mathrm{CDR}) \leq 0.65[8]$.

Moderate glaucoma: optic nerve abnormalities consistent with glaucoma and glaucomatous visual field abnormalities in one hemifield and not within $5^{\circ}$ of fixation with moderate glaucomatous disc features of vertical CDR $=0.7-0.85$ [8].

Advanced glaucoma: patients who have a CDR of 0.85-0.95 and glaucomatous visual field abnormalities in both hemifields and/or loss within $5^{\circ}$ of fixation in at least one hemifield, with the ability of light perception [8].

\subsection{Method of Data Analysis}

2.6.1. Linear Mixed Model (LMM). Longitudinal data are the data of repeated measurements at a limited number of time points with predetermined designs on a time scale, time interval, and other related conditions [9]. Therefore, to know the evolution of the disease of glaucoma, we have to incorporate the LMM. The LMM is widely used in which random effects are introduced to incorporate the betweensubject variation and within-subject correlation in the data. The random effects determine the correlation structure between observations on the same subject, and they take account of heterogeneity among repeated measures due to unobserved characteristics.

The general linear mixed-effect model is defined as follows [10]:

$$
y_{i}=X_{i} \beta+Z_{i} b_{i}+\varepsilon_{i}
$$

where $y_{i}$ is the $\left(n_{i} \times 1\right)$ vector of repeated measurements for the $i^{\text {th }}$ subject, $\beta$ is a $p \times 1$ vector of the fixed effects parameters, $X_{i}$ is an $n_{i} \times p$ known design matrix corresponding to fixed effects $\beta, b_{i}$ is a $q \times 1$ vector of randomeffect parameters, $Z_{i}$ is an $n_{i} \times q$ known design matrix corresponding to random effects $b_{i}$, and $\varepsilon_{i}$ is the $\left(n_{i} \times 1\right)$ vector of the error terms.

Step-wise linear mixed model analysis with forwarding selection and backward elimination with removal as well as inclusion probability of $5 \%$ was used to identify the independent predictors of longitudinally measured IOP of 
glaucoma patients. All the significant variables under univariate analysis were considered as candidate variables for multivariable analysis. All the results were interpreted at a $5 \%$ level of significance and a 95\% confidence level. $R$ 4.0.3 was used for the statistical analysis.

\section{Results}

The descriptive results of glaucoma patient characteristics from the ophthalmology clinic medical charts at FHRH are shown in Tables 1 and 2.

3.1. Assessment of Normality Assumption. The normal Q-Q plot of the original data seems to satisfy the assumption of normality. However, the normal Q-Q plot for the logtransformed data shows that the normality assumption was violated (Figure 1).

We could observe that the histogram of the original data seems to satisfy the assumption of normality. However, for the histogram of the log-transformed data, the assumption of normality was violated (Figure 2).

3.2. Individual and Mean Profile Analysis. The individual profile plot was obtained to gain some insights into the data or to show the pattern of the data over time. Though, the pattern of the overall individual plots of IOP measurements of patients over time demonstrates the variability within and between patients in IOP over time (at 60 months) in this study. Since the measurements were not equally spaced across the different subjects and data are not balanced, the LOESS smoothing technique was used instead (Figure 3).

The red line, LOESS smoothing technique (Figure 4), suggests that the mean structure of the IOP is nearly linear over time (i.e., the relationship between IOP and follow-up time seems to be linear), and the mean IOP decreased over time.

\subsection{Selection of Covariance Structure in a Linear Mixed Model.}

For the LMM to be valid, the covariance among repeated measures must be modeled properly. To identify the appropriate covariance structure, the three commonly used covariance structures, compound symmetry (CS), first-order autoregressive (AR (1)), and unstructured (UN), were considered [11].

The first-order autoregressive (AR (1)) covariance structure was selected due to the smallest AIC and BIC values compared to the remaining covariance structures (Table 3).

3.4. Selection of Random Effects in a Linear Mixed Model. By using a first-order autoregressive (AR (1)) covariance structure, we implemented different linear mixed models to study the longitudinal outcome by including the subjectspecific random effects. Finally, we compared the information criteria values for the selection of random effects to be included in the linear mixed-effect model. Choosing the random intercept and slope model allows the intercept and coefficient to vary randomly among individuals. That means the individual IOP of glaucoma patients varies from visit to visit randomly. Therefore, the random intercept and slope were better for the data for the linear mixed-effect model based on its lower values of AIC and BIC (Table 4).

3.5. Univariable Analysis for the Linear Mixed Model. Based on the univariable analysis, all predictors were the candidate variables for multivariable analysis of linear mixed models at a $25 \%$ level of significance.

3.6. Multivariable Analysis for Linear Mixed Model. The results of the multivariable analysis are presented in Table 5. The estimated coefficient of fixed-effect intercept was 25.18 ( $p$ value $<0.001$ ). For a year increased in age, the average IOP of the patients was significantly increased by $0.07 \mathrm{mmHg}$ ( $p$ value $=0.001)$ keeping all variables constant. The average IOP of the patients who live in urban areas was significantly lower by $1.6 \mathrm{mmHg}(p$ value $=0.011)$ compared to the patients who live in rural areas, keeping all other variables constant. The average IOP of the patients who had family history with glaucoma was significantly higher by $4.9 \mathrm{mmHg}$ ( $p$ value $<0.001$ ) compared to the patients who had no family history with glaucoma.

The average IOP of the patient who takes timolol and pilocarpine medication was significantly lower by $2.02 \mathrm{mmHg}(p$ value $=0.047)$ compared to the patient who takes timolol medication. The average IOP of the patients who had greater than 0.7 cup-disk ratios was significantly higher by $2.6 \mathrm{mmHg}$ ( $p$-value $<0.001)$ compared to the patient who had less than or equal to 0.7 cup-disk ratios. For a unit increased in visits (follow-up times), the average IOP of glaucoma patients was significantly decreased by $0.34 \mathrm{mmHg}$ ( $p$ value $<0.001)$.

The variability between patients was $31.12 \mathrm{mmHg}$, while the variability between patients was $0.2 \mathrm{mmHg}$, the correlation between intercept and slope was -0.78 , and the variability within patients was $42.72 \mathrm{mmHg}$.

\section{Discussion}

The main objective of this study was to identify factors that affect longitudinal intraocular pressure change of glaucoma patients at Felege Hiwot Referral Hospital, Bahir Dar, Ethiopia. In this study, the linear mixed-effect model for longitudinal IOP was used. The assumption of normality was checked using the normal Q-Q plot and histogram by comparing the plot of original and log-transformed data of glaucoma patients. All plots of the original data indicate that there is no deviation from the normality assumption and no need for transformation. The mean of the longitudinal IOP was linearly decreasing over time. The data were analyzed using the linear mixed-effect model by incorporating subject-specific variability. In this study, the first-order autoregressive (AR (1)) covariance structure and random intercept and slope model had a smaller AIC and BIC compared to other models. 
TABLE 1: Baseline characteristics of categorical variables of glaucoma patients.

\begin{tabular}{|c|c|c|c|}
\hline Variables & Categories & No. of patients (\%) & Mean IOP \\
\hline \multirow{2}{*}{ Gender } & Female & $108(32.9)$ & 28.29 \\
\hline & Male & $220(67.1)$ & 29.92 \\
\hline \multirow{2}{*}{ Place of residence } & Rural & $146(44.5)$ & 30.90 \\
\hline & Urban & $182(55.5)$ & 28.05 \\
\hline \multirow{2}{*}{ Family history with glaucoma } & Yes & $98(29.9)$ & 35.16 \\
\hline & No & $230(70.1$ & 27.09 \\
\hline \multirow{2}{*}{ Family support } & Yes & $280(85.4)$ & 28.65 \\
\hline & No & $48(14.6)$ & 33.71 \\
\hline \multirow{4}{*}{ Type of medication } & Timolol & $65(19.8)$ & 29.98 \\
\hline & Timolol and pilocarpine & $82(25.0)$ & 27.67 \\
\hline & Timolol and diamox & $111(33.8)$ & 29.66 \\
\hline & Timolol, pilocarpine, and diamox & $70(21.3)$ & 30.60 \\
\hline \multirow{3}{*}{ Duration of treatment } & Less than 2 years & $135(41.2)$ & 30.08 \\
\hline & $2-5$ years & $106(32.3)$ & 30.81 \\
\hline & Above 5 years & $87(26.3)$ & 27.51 \\
\hline \multirow{3}{*}{ Stage of glaucoma } & Early & $121(36.9)$ & 26.95 \\
\hline & Moderate & $52(15.9)$ & 28.86 \\
\hline & Advanced & $155(47.3)$ & 31.84 \\
\hline \multirow{2}{*}{ Cup-disk ratio } & Less than or equal to 0.7 & $173(52.7)$ & 26.84 \\
\hline & Greater than 0.7 & $153(47.3)$ & 32.59 \\
\hline
\end{tabular}

TABLE 2: Baseline characteristics of continuous variables of glaucoma patients $(n=328)$.

\begin{tabular}{lcccc}
\hline Variables & Minimum & Maximum & Mean & Std.dev \\
\hline IOP in mmHg & 9.50 & 51.70 & 31.09 & 9.54 \\
Age in years & 24 & 89 & 55.86 & 13.35 \\
\hline
\end{tabular}

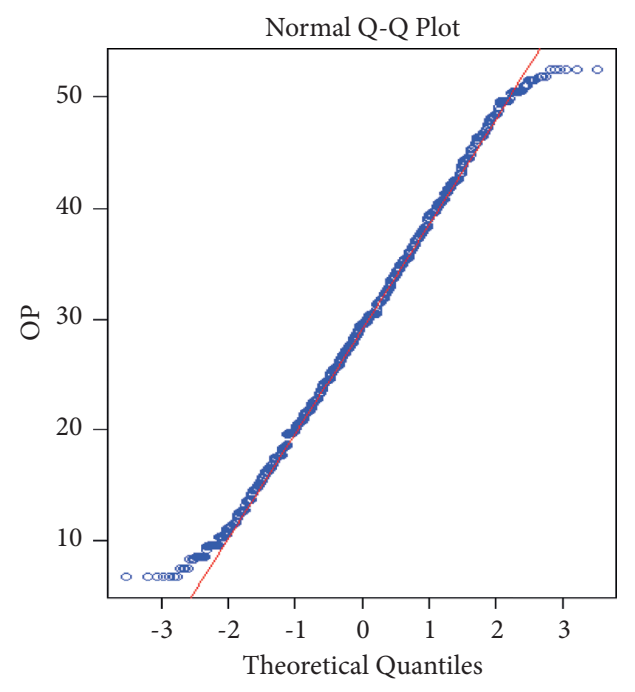

FIGURE 1: Normal Q-Q plot of original IOP

Age is an important sociodemographic predictor of IOP. Higher age significantly increased the IOP of glaucoma patients. This finding was consistent with another study [12-14]. This might be older people who live with ocular hypertension due to this reason increase IOP in older age. The average IOP of glaucoma patients among urban residences was 1.6 lower as compared to those who were rural residences. This is similar to the study conducted at Gondar University Hospital, Ethiopia

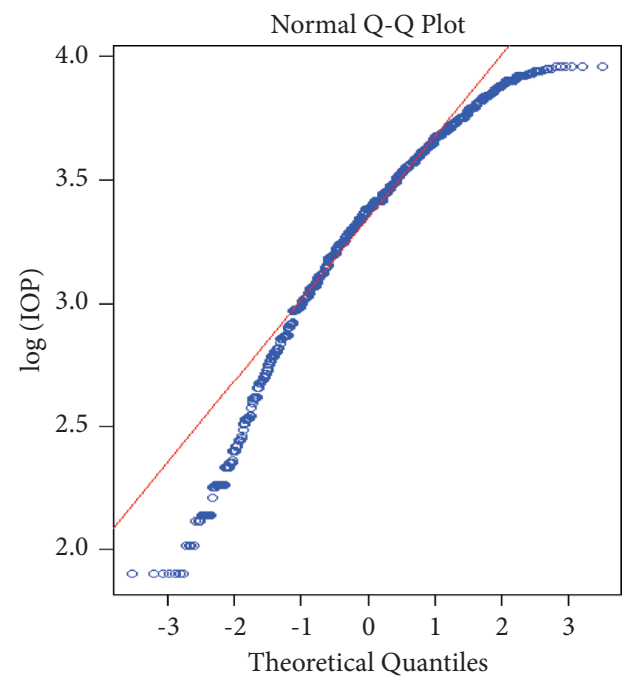

(a) and $\log$ of IOP (b) for glaucoma patients.

[15]. This might be explained by patients who were urban residents having better awareness and knowledge on glaucoma and medication of IOP as compared to rural residents.

The mean IOP was found to evolve differently between patients with family history of glaucoma and patients without family history of glaucoma based on the result of linear mixed models. The average IOP was higher for patients who had family history of glaucoma compared to 

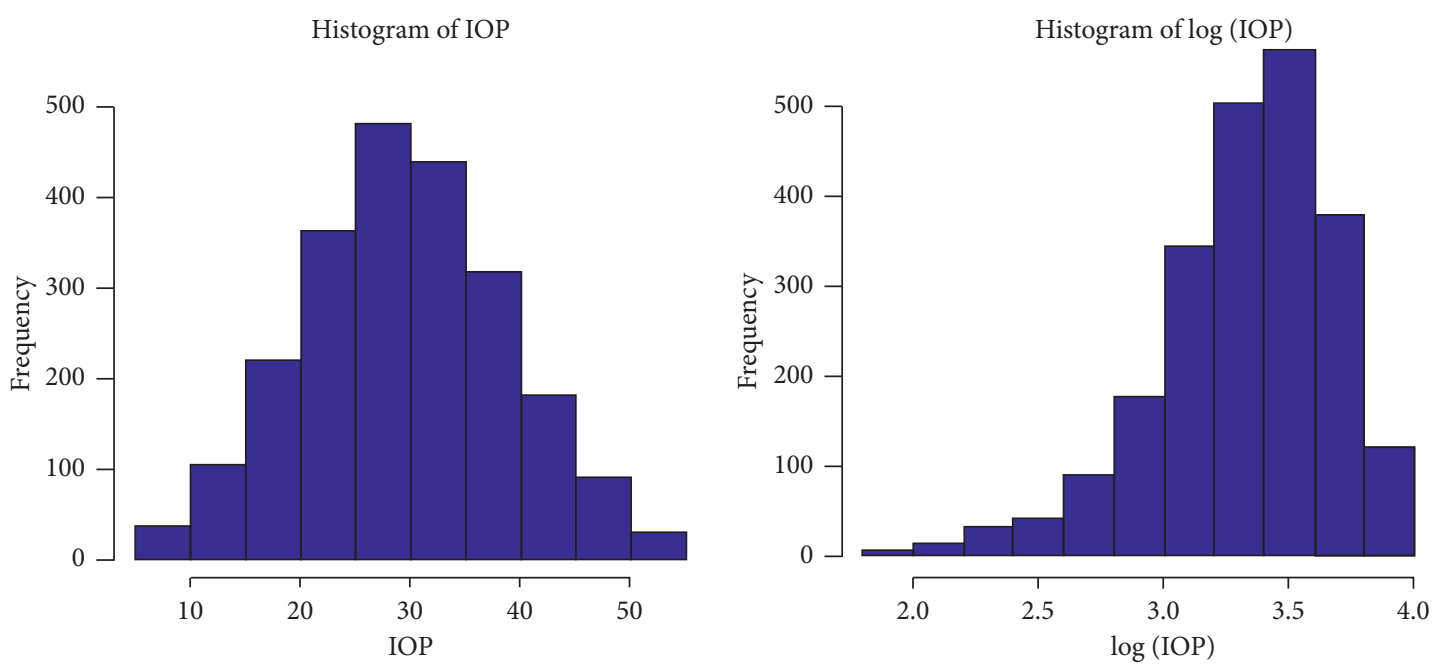

FIGURE 2: Histogram of original IOP (a) and log of IOP (b) for glaucoma patients.

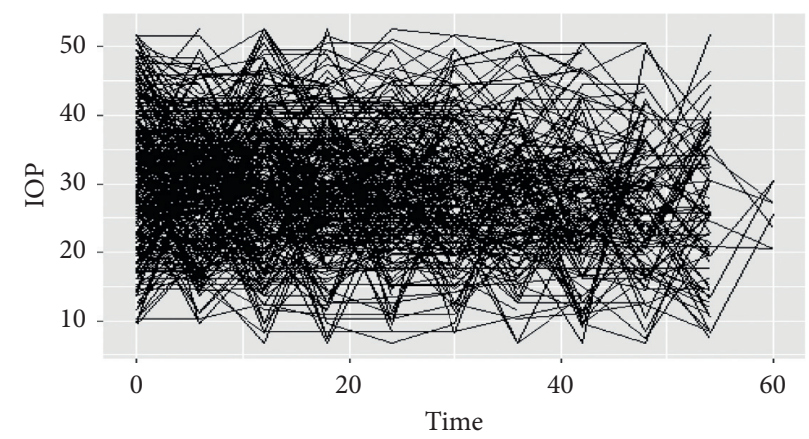

FIgURE 3: Individual profile plot for IOP of glaucoma patients.

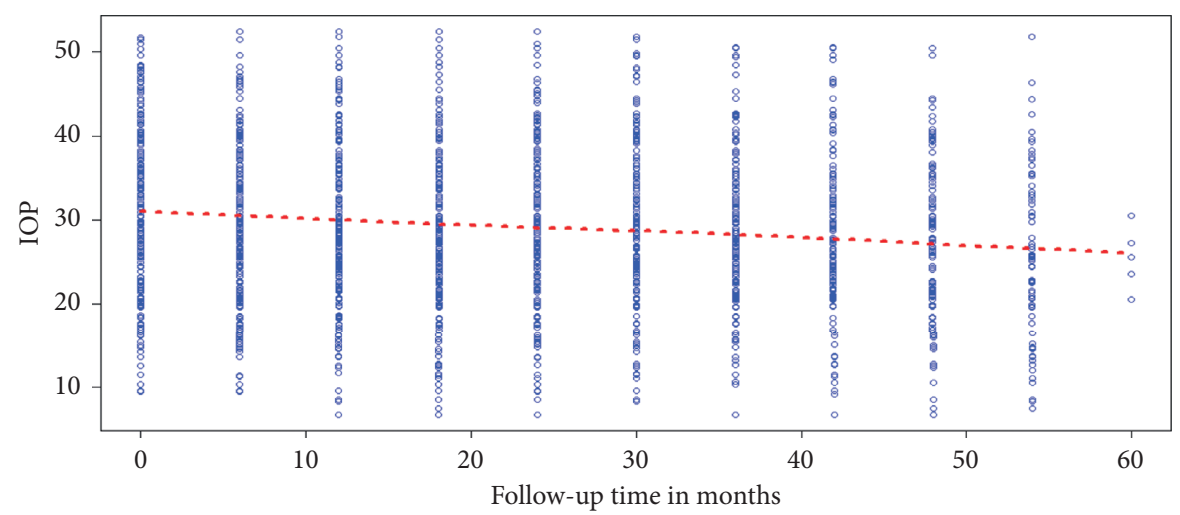

FIgURE 4: Loess smoothing plot with the average trend line of IOP of glaucoma patients.

patients who had no family history of glaucoma. This result was consistent with the other studies $[16,17]$. This might be due to the fact that the family history of glaucoma is genetically transformed to their child. Type of medication was significantly associated with the IOP of glaucoma patients. This finding showed that the glaucoma patients who taketimolol and pilocarpine medication reduce IOP. This finding was consistent with another study [18], and the result showed that the use of any class of glaucoma medication was associated with a statistically significant to reduced IOP of glaucoma patients. This might be the type of glaucoma medication that has a similar effect for glaucoma patients.

The average IOP of glaucoma patients who had a cup-disk ratio greater than 0.7 was higher compared to that of patients who had a cup-disk ratio less than or equal to 0.7 . This finding was consistent with another study [19], and the result showed an increased IOP of glaucoma patients in a larger cup-disc 
TABLE 3: Comparison of covariance structure for the linear mixed-effect model.

\begin{tabular}{lccc}
\hline Covariance structure & AIC & BIC & Log-likelihood \\
\hline CS & 15528.87 & 15620.52 & -7748.43 \\
AR (1) & $\mathbf{1 5 5 2 6 . 7 2}$ & $\mathbf{1 5 6 1 8 . 3 7}$ & $-\mathbf{7 7 4 7 . 3 6}$ \\
UN & 15526.87 & 15619.79 & -7748.43 \\
\hline
\end{tabular}

TABLE 4: Selection of random effects to be included in the LMM.

\begin{tabular}{lccc}
\hline Models for random effect & AIC & BIC & LogLik \\
\hline Random intercept & 15531.24 & 15621.43 & -7751.62 \\
Random slope & 15657.57 & 15737.76 & -7814.78 \\
Random intercept and slope & $\mathbf{1 5 5 2 6 . 7 2}$ & $\mathbf{1 5 6 1 8 . 3 7}$ & $-\mathbf{7 7 4 7 . 3 6}$ \\
\hline
\end{tabular}

TABle 5: Result of the final linear mixed model for glaucoma patients.

\begin{tabular}{|c|c|c|c|c|c|c|}
\hline \multirow{2}{*}{ Covariates } & \multirow{2}{*}{ Estimate } & \multirow{2}{*}{ Std. error } & \multicolumn{2}{|c|}{$95 \% \mathrm{CI}$} & & \multirow{2}{*}{$p$ value } \\
\hline & & & Lower & Upper & & \\
\hline Intercept & 25.18 & 1.30 & 22.64 & 27.73 & & $<0.001$ \\
\hline Age & 0.07 & 0.02 & 0.03 & 0.11 & & 0.001 \\
\hline \multicolumn{7}{|l|}{ Residence $($ ref $=$ rural $)$} \\
\hline Urban & -1.60 & 0.63 & -2.84 & -0.36 & & 0.011 \\
\hline \multicolumn{7}{|l|}{ Family history with glaucoma $($ ref $=$ no $)$} \\
\hline Yes & 4.90 & 0.78 & 3.38 & 6.43 & & $<0.001$ \\
\hline \multicolumn{7}{|l|}{ Type of medication $($ ref $=$ timolol $)$} \\
\hline Timolol and pilocarpine & -2.02 & 1.02 & -4.01 & -0.03 & & 0.047 \\
\hline Timolol and diamox & -0.03 & 1.10 & -2.18 & 2.13 & & 0.982 \\
\hline Timolol, pilocarpine, and diamox & 0.90 & 1.21 & -1.47 & 3.26 & & 0.109 \\
\hline \multicolumn{7}{|l|}{ Cup-disk ratio $($ ref $=\leq 0.7)$} \\
\hline Greater than 0.7 & 2.60 & 0.69 & 1.24 & 3.96 & & $<0.001$ \\
\hline Visits & -0.34 & 0.07 & -0.47 & -0.21 & & $<0.001$ \\
\hline \multirow{2}{*}{ Random effect } & Std dev & \multicolumn{5}{|c|}{$95 \% \mathrm{CI}$} \\
\hline & Std. dev & & & & Upper & \\
\hline Intercept $\left(b_{0 i}\right)$ & 5.58 & & & & 6.50 & \\
\hline Visits $\left(b_{1 i}\right)$ & 0.50 & & & & 0.76 & \\
\hline Correlation $\left(b_{0 i}, b_{1 i}\right)$ & -0.57 & & & & -0.31 & \\
\hline Residual $\left(\varepsilon_{i}\right)$ & 6.54 & & & & 6.79 & \\
\hline
\end{tabular}

ratio $>0.7$. The probable explanation is when cup-disk ratio increased, IOP also increased due to the nature of the disease.

\section{Conclusions}

In conclusion, this study was a five-year (January 2014 to December 2018) retrospective cohort study based on 328 glaucoma patients attending ophthalmologic clinics at Felege Hiwot Referral, Bahir Dar, Ethiopia. The linear mixed model analysis showed that the predictor age, residence, family history of glaucoma, type of medication, cup-disk ratio, and follow-up time (visits) were significantly associated with the average IOP. Therefore, healthcare providers should give due attention and prioritize those identified factors and give frequent counseling about reducing intraocular pressure of primary open-angle glaucoma patients.

\section{Abbreviations}

AIC: Akaike information criterion

BIC: Bayesian information criterion
IOP: Intraocular pressure

LMM: Linear mixed model

FHRH: Felege Hiwot Referral Hospital.

\section{Data Availability}

The datasets used and/or analyzed during the current study are available from the corresponding author on reasonable request.

\section{Ethical Approval}

The Bahir Dar University Ethical Review Board approved the study as per the Declaration of Helsinki. Ethical clearance was obtained from Bahir Dar University, College of Science, Ethical Review Committee. The individual patients were not subject to any harm as far as confidentiality was kept.

\section{Conflicts of Interest}

The authors declare no conflicts of interest. 


\section{Authors' Contributions}

The first author wrote the proposal, developed the data collection format, supervised the data collection process, and analyzed and interpreted the data. The second author participated in the design, critically read the manuscript, and gave constructive comments for the betterment of the manuscript. All authors read and approved the manuscript.

\section{Acknowledgments}

The authors acknowledge the Amhara National Regional State Health Bureau and Ophthalmic Clinic at Felege Hiwot Referral Hospital, Ethiopia, for the data they supplied for this research.

\section{References}

[1] B. M. Davis, L. Crawley, M. Pahlitzsch, F. Javaid, and M. F. Cordeiro, "Glaucoma: the retina and beyond," Acta Neuropathologica, vol. 132, no. 6, pp. 807-826, 2016.

[2] K. A. Durowade, T. M. Akande, O. I. Musa et al., "Prevalence and risk factors of glaucoma among adults in rural and urban communities of Ilorin west local government area, NorthCentral Nigeria," International Journal of Clinical Medicine Research, vol. 3, no. 1, pp. 6-12, 2016.

[3] N. M. Farandos, A. K. Yetisen, M. J. Monteiro, C. R. Lowe, and S. H. Yun, "Contact lens sensors in ocular diagnostics," Advanced healthcare materials, vol. 4, no. 6, pp. 792-810, 2015.

[4] Y. K. Song, C.-K. Lee, J. Kim, S. Hong, C. Y. Kim, and G. J. Seong, "Instability of 24-hour intraocular pressure fluctuation in healthy young subjects: a prospective, crosssectional study," BMC Ophthalmology, vol. 14, no. 1, p. 127, 2014.

[5] D. Bruce and V. M. Eshun, "Psychological experience of clients diagnosed with glaucoma in two selected eye clinics in Accra, Ghana," International Journal of Regulation and Governance, vol. 5, no. 9, pp. 52-67, 2017.

[6] Y. Berhane, A. Worku, A. Bejiga et al., "Prevalence and causes of blindness \& low vision in Ethiopia," The Ethiopian Journal of Health Development, vol. 21, pp. 204-210, 2007.

[7] A. T. Giorgis, "Raising public awareness of glaucoma in Ethiopia," Community Eye Health, vol. 25, p. 46, 2012.

[8] A. S. Assem, A. S. Fekadu, A. A. Yigzaw, Z. M. Nigussie, and A. A. Achamyeleh, "Level of glaucoma drug adherence and its associated factors among adult glaucoma patients attending Felege Hiwot specialized hospital, Bahir Dar City, Northwest Ethiopia," Clinical Optometry, vol. 12, p. 189, 2020.

[9] L. Xian, "Introduction to longitudinal data analysis in psychiatric research," Shanghai Archives of Psychiatry, vol. 27, p. 256, 2015.

[10] D. Rizopoulos, Joint Models for Longitudinal and Time-ToEvent Data: With Applications in R, Chapman and Hall/CRC, Boca Raton, FL, USA, 2012.

[11] J. Cheng, L. J. Edwards, M. M. Maldonado-Molina, K. A. Komro, and K. E. Muller, "Real longitudinal data analysis for real people: building a good enough mixed model," Statistics in Medicine, vol. 29, no. 4, pp. 504-520, 2010.

[12] L. Rossetti, M. Digiuni, M. Giovanni et al., "Blindness and glaucoma: a multicenter data review from 7 academic eye clinics," PLoS One, vol. 10, no. 8, Article ID e0136632, 2015.
[13] L. Tamrat, G. W. Gessesse, and Y. Gelaw, "Adherence to topical glaucoma medications in Ethiopian patients," Middle East African Journal of Ophthalmology, vol. 22, pp. 59-63, 2015.

[14] F. A. Ayele, B. Zeraye, Y. Assefa, K. Legesse, T. Azale, and M. J. Burton, "The impact of glaucoma on quality of life in Ethiopia: a case-control study," BMC Ophthalmology, vol. 17, no. 1, p. 248, 2017.

[15] D. H. Anbesse, B. T. Yibekal, and N. L. Assefa, "Adherence to topical glaucoma medications and associated factors in gondar university hospital tertiary eye care center, northwest Ethiopia," European Journal of Ophthalmology, vol. 29, no. 2, pp. 189-195, 2019.

[16] X. Han, T. Yang, J. Zhang et al., "Longitudinal changes in intraocular pressure and association with systemic factors and refractive error: lingtou eye cohort study," BMJ Open, vol. 8, no. 2, Article ID e019416, 2018.

[17] D. Zhao, M. H. Kim, R. Pastor-Barriuso et al., "A longitudinal study of association between adiposity markers and intraocular pressure: the Kangbuk Samsung Health Study," PLoS One, vol. 11, no. 1, Article ID e0146057, 2016.

[18] D. D. French and C. E. Margo, "Glaucoma medications and mortality: a retrospective cohort study," Annals of Epidemiology, vol. 20, no. 12, pp. 917-923, 2010.

[19] S. K. Gardiner, C. A. Johnson, and S. Demirel, "Factors predicting the rate of functional progression in early and suspected glaucoma," Investigative Opthalmology \& Visual Science, vol. 53, no. 7, pp. 3598-3604, 2012. 